\title{
Expression of the ERM family members (ezrin, radixin and moesin) in breast cancer
}

\author{
HERMAN FERNANDO, TRACEY A. MARTIN, ANTHONY DOUGLAS-JONES, \\ HOWARD G. KYNASTON, ROBERT E. MANSEL and WEN G. JIANG
}

\begin{abstract}
Metastasis and Angiogenesis Research Group, Wales College of Medicine, Cardiff University, Cardiff, UK
\end{abstract}
Received February 3, 2009; Accepted March 19, 2009

DOI: 10.3892/etm_00000025

\begin{abstract}
The ERM family is composed of the proteins ezrin, moesin and radixin, which are cell structure-related proteins. Despite the detection of viable roles of ERM family proteins, the impact of these molecules in cancer pathogenesis has yet to be investigated. Evidence emerging from clinical and translational studies showed that the ERM family is linked to disease progression in clinical cancers. We aimed to establish the pattern of expression of the ERM proteins and deduce a possible relationship between these molecules and clinical outcome in a cohort of human breast cancers. The expression of the three ERM molecules at the mRNA and protein levels in a cohort of 122 human breast cancers and 32 normal breast tissues were analysed and correlated with pathological and clinical information as well as patient outcome. The three molecules were positively stained in mammary tissues while the staining pattern was lost in the malignant cells. Low levels of moesin and radixin transcripts were seen in tumours from patients with metastasis, local recurrence and in patients who succumbed to the disease (moesin: $\mathrm{p}=0.039, \mathrm{p}=0.037$ and $\mathrm{p}=0.066$, respectively, and radixin: $\mathrm{p}=0.039, \mathrm{p}=0.039$ and $\mathrm{p}=0.04$, respectively). Ezrin levels were significantly lower in tumour recurrence and in patients who succumbed to the disease ( $p=0.0001$ and $p=0.59$, respectively). Using the Kaplan-Meier survival analysis, a general trend of higher levels of ERM was observed, with marginal long overall and disease-free survival. In conclusion, an inverse relationship between ERM expression and tumour behaviour of breast cancer patients was noted. However, further work needs to be conducted in other types of cancer in clinical situations to obtain consistent results.
\end{abstract}

Correspondence to: Professor Wen G. Jiang, Metastasis and Angiogenesis Research Group, Cardiff University, School of Medicine, Heath Park, Cardiff CF4 4XN, UK

E-mail: jiangw@cf.ac.uk

Key words: ERM family, ezrin, moesin, radixin, cell adhesion, cytoskeletal proteins, breast cancer, metastasis, survival

\section{Introduction}

The ERM family is composed of the proteins ezrin, moesin and radixin, which are cell structure-related proteins (1). Their sequence of the amino-terminal halves (300 amino acids) is highly conserved and is found in band 4.1, an erythrocyte membrane protein. Together the proteins are called the band 4.1 superfamily and their common domain is referred to as the FERM domain, in combination with merlin/schwannomin, the NF2 tumour suppressor protein and Talin. These highly homologous proteins constitute a family with structural and viable relationships. Moreover, the proteins are localized to the submembranous cytoskeleton and are traditionally known as molecules responsible for maintaining cell integrity and morphology. ERM proteins are also involved in the binding process between the plasma membrane and the actin cytoskeleton, and are involved in a variety of cellular functions, such as cell adhesion, migration and the organization of cell surface structures.

It has been suggested that radixin is involved in anchoring the 'pointed' ends of actin filaments to the membrane in stereocilia (2). Using antigen-activated T cells, ERM proteins regulate cytoskeleton relaxation and promote $\mathrm{T}$ cell-APC (antigen-presenting cell) conjugation. Ezrin-radixin-moesin (ERM) proteins are rapidly inactivated through a VAV1-RAC1 pathway (3). The resulting disanchoring of the cortical actin cytoskeleton from the plasma membrane decreased cellular rigidity, resulting in a more efficient $\mathrm{T}$ cell-APC conjugate formation. Thus, this pathway favours immunological synapse formation and the development of an effective immune response.

Ezrin was shown to act as an anchorage protein for CD44, a cell adhesion molecule that is widely involved in metastatic cells, as well as ICAM2 (4). We reported that ezrin interacts with the cadherin complex and co-ordinates the cell-cell adhesion mechanisms in epithelial cells (5). Thus, the above studies showed that the disruption or deletion of ezrin and its family members disrupted homotypic and heterotypic cell-cell adhesion. ERM protein functions, and their link to CD44 and ICAM2, suggest a potential role of the ERM family in cancer invasion and metastasis.

Despite the detection of the viable roles of ERM family proteins, the impact of these molecules in cancer pathogenesis has yet to be investigated. Evidence emerging from clinical 
and translational studies showed that the ERM family is linked to disease progression in clinical cancers. For example, in patients with head and neck squamous cell carcinoma, high levels of ezrin protein staining were associated with a shorter survival (6). In prostate tissues, ezrin immunoreactivity is associated with the Gleason score and seminal vesicle invasion of the patients (7). In paediatric soft tissue sarcoma (STS), high levels of ezrin immunoreactivity are associated with disease-free and overall survival of the patients (8). Using a tissue microarray of $>5000$ tumours, Bruce et al (9) have shown that a link exists between ezrin and clinical outcome in patients with a variety of tumour types.

In the present study, we aimed to establish the pattern of expression of the ERM proteins and deduce a possible relationship between these molecules and clinical outcome in a cohort of human breast cancers.

\section{Materials and methods}

Materials. RNA extraction and RT kits were obtained from AbGene Ltd., Surrey, England, UK. PCR primers were designed using Beacon Designer (CA, USA) and synthesised by Invitrogen Ltd. (Pasley, Scotland, UK). Molecular biology grade agarose and DNA ladder were purchased from Invitrogen (Pasley). Master mix for routine PCR and customised master mix for quantitative PCR were from AbGene. Polyclonal antibody to human moesin and radixin, and mouse monoclonal antibody to human ezrin were purchased from Santa Cruz Biotechnology Ltd. (Santa Cruz, CA, USA) and Affinity Antibodies, Inc. (Exeter, England, UK), respectively. Anti-ER (543) and anti-ER- $\beta$ (8974) antibodies were purchased from Santa Cruz Biotechnology, Inc. Peroxidase conjugated anti-rabbit and anti-goat antibodies were from Sigma and a biotin universal staining kit was from Vector Laboratories (Nottingham, England, UK).

Cells and tissue samples. Breast cancer tissues $(\mathrm{n}=120)$ and normal background tissues $(\mathrm{n}=32)$ were collected immediately after surgery and stored in the deep freezer until use. Details of histology and clinical outcome were obtained from pathology reports as was previously reported (Table I). Patients were routinely followed after surgery. The median follow-up period was 120 months. The presence of tumour cells in the collected tissues was verified by a consultant pathologist who examined H\&E-stained frozen sections $(10,11)$.

Tissue processing, RNA extraction, cDNA synthesis and $R T-P C R$. Frozen sections of tissues were cut at 5-10 $\mu \mathrm{m}$ and were kept for immunohistochemistry and routine histology (12). A further 15-20 sections were mixed and homogenised using a hand-held homogeniser, in ice-cold RNA extraction solution. RNA concentration was determined using a UV spectrophotometer. Reverse transcription was carried out using a RT kit with an anchored oligo(dt) primer supplied by AbGene, and 1 pg total RNA in a 96-well plate. The quality of cDNA was verified using $\beta$-actin primers.

Quantitative analysis of ezrin, radixin and moesin transcripts. The level of ERM transcripts from the cDNA prepared as above was determined using a real-time quantitative PCR,
Table I. Clinicopathological details of the study cohort.

\begin{tabular}{|c|c|}
\hline Clinical information & No. \\
\hline \multicolumn{2}{|l|}{ Nodal status } \\
\hline Negative & 65 \\
\hline Positive & 55 \\
\hline \multicolumn{2}{|l|}{ Grade } \\
\hline 1 & 23 \\
\hline 2 & 41 \\
\hline 3 & 56 \\
\hline \multicolumn{2}{|l|}{ Histology } \\
\hline Ductal & 94 \\
\hline Lobular & 14 \\
\hline Medullary & 2 \\
\hline Tubular & 2 \\
\hline Mucinous & 4 \\
\hline Other & 4 \\
\hline \multicolumn{2}{|l|}{ TNM staging } \\
\hline TNM 1 & 69 \\
\hline TNM 2 & 40 \\
\hline TNM 3 & 7 \\
\hline TNM 4 & 4 \\
\hline \multicolumn{2}{|l|}{ Clinical outcome } \\
\hline Disease-free & 81 \\
\hline Metastasis & 7 \\
\hline Local recurrence & 5 \\
\hline Succumbed to breast cancer & 20 \\
\hline Succumbed to unrelated disease & 7 \\
\hline
\end{tabular}

based on the Amplifluor ${ }^{\mathrm{TM}}$ technology as previously reported $(13,14)$, which has been modified from a method previously described (13). Briefly, PCR primer pairs were designed using the Beacon Designer software (version 2, CA, USA) (sequence given in Table II). An additional sequence, known as the $\mathrm{Z}$ sequence (5'actgaacctgaccgtaca'3), which is complementary to the universal Z probe (13) (Intergen Inc., England, UK), was added to one of the primers. A Taqman detection kit for $\beta$-actin was purchased from Perkin-Elmer. The reaction was carried out using hot-start Q-master mix (Abgene), 10 pmol of specific forward primer, 10 pmol reverse primer which has the $\mathrm{Z}$ sequence, 100 pmol of 6-carboxyfluorescein FAM-tagged probe (Intergen Inc.) and cDNA from 50 ng RNA. The reaction was carried out using Icycler IQ ${ }^{\mathrm{TM}}$ (Bio-Rad) which is equipped with an optic unit that allows real-time detection of 96 reactions, using the conditions: $94^{\circ} \mathrm{C}$ for $12 \mathrm{~min}, 50$ cycles of $94^{\circ} \mathrm{C}$ for $15 \mathrm{sec}, 55^{\circ} \mathrm{C}$ for $40 \mathrm{sec}$ and $72^{\circ} \mathrm{C}$ for $20 \mathrm{sec}$ $(13,15,16)$. Primers used for quantification of the oestrogen receptor (ER) and ER- $\beta$ were as previously reported (17). Cytokeratin-19 (CK19) was used to compare cellularity during the analysis (18). Transcript levels were generated from an internal standard (12) that was simultaneously amplified with the samples. The transcript levels were based on equal amounts of RNA and as a target/CK19 ratio. 
Table II. Primer sequences (underlined sections are the Z-sequence for quantification).

\begin{tabular}{|c|c|c|}
\hline & Sense (5'-'3) & Antisense (5'-'3) \\
\hline Ezrin & agatgagcagtctgccttt & actgaacctgaccgtacacaacatgagagattgggaaaga \\
\hline Moesin & cgacagaagaaggagagtga & Actgaacctgaccgtacaggtgtactcatggcagtctt \\
\hline Radixin & gaccagatgaagaatcagga & Actgaacctgaccgtacaagttgettccetcttcctttt \\
\hline CK19 quantification & caggtccgaggttactgac & actgaacctgaccgtacacactttctgccagtgtgtcttc \\
\hline ER & cctactacctggagaacgag & Ctcttcggtcttttcgtatg \\
\hline $\mathrm{ER}-\beta$ & aaaagaatcattcaatgaca & Attaacacctccatccaaca \\
\hline$\beta$-actin & atgatatcgccgegctcg & cgctcgtgtaggatcttca \\
\hline
\end{tabular}
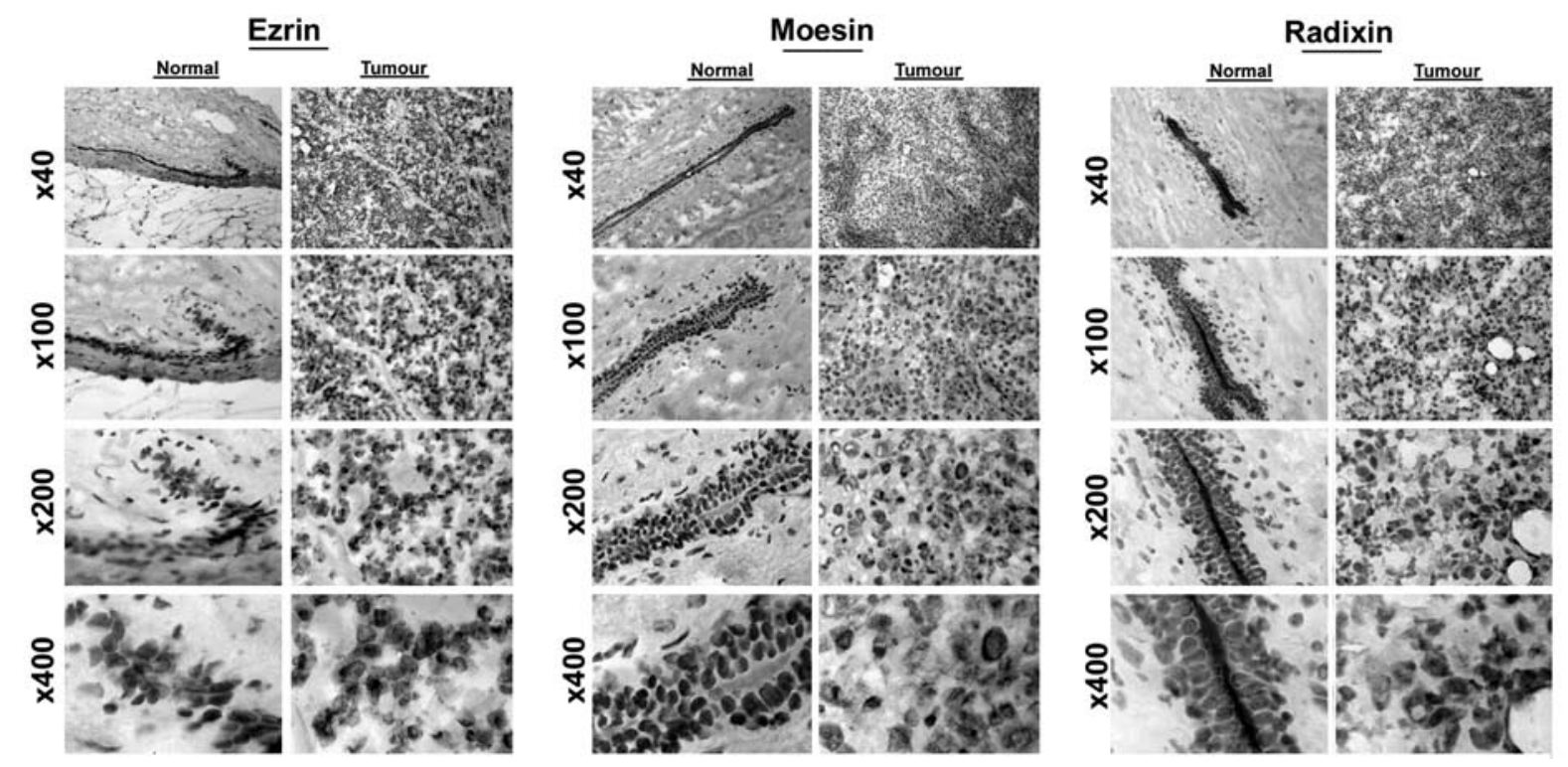

Figure 1. Immunohistochemical staining of ezrin (left panel), moesin (middle panel) and radixin (right panel) in normal mammary tissue (left column in the respective panel) and breast tumour tissue (right in the respective panel). Staining is shown at varying magnifications.

Immunohistochemical staining of ezrin, radixin and moesin. Immunohistochemical staining was based on a method described earlier (16). Frozen sections of breast tumours and background tissues were cut at $6 \mu \mathrm{m}$ using a cryostat. The sections were mounted on Superfrost plus microscope slides, air dried and fixed in a mixture of $50 \%$ acetone and $50 \%$ methanol. The staining procedure was completed simultaneously for the section, to ensure comparison. The sections were then placed in Optimax wash buffer for 5-10 min to rehydrate. Sections were incubated for $20 \mathrm{~min}$ in a $0.6 \%$ BSA blocking solution and probed with the primary antibody (diluted at 1:100 for anti-moesin and anti-radixin and 1:50 for anti-ezrin). Following extensive washings, sections were incubated for $30 \mathrm{~min}$ in the secondary biotinylated antibody (Multilink Swine anti-goat/mouse/rabbit immunoglobulin, Dako Inc.). Following washings, the Avidin-Biotin Complex (Vector Laboratories) was applied to the sections followed by extensive washings. Diaminobenzidine chromogen (Vector Laboratories) was added to the sections which were incubated in the dark for $5 \mathrm{~min}$. Sections were counterstained in Gill's haematoxylin and dehydrated in ascending grades of meth- anol before they were cleared in xylene and mounted under a cover slip. The staining intensity of the respective proteins in the cytoplasmic area and nucleus was semi-quantified from non-counterstained images, using a method established in our laboratory $(18,19)$. Briefly, grayscale digitized images were imported into the Optimas software (Optimas 6.0). The staining intensity was analysed in the nucleus and cytoplasmic compartments. Control staining (without primary antibody) was used for extraction of the background staining. Intensity data were exported to Excel for statistical analysis and are shown here as the mean intensity of either the cytoplasmic region or nucleus staining.

Statistical analysis was carried out using the MannWhitney $U$ and Kruskal-Wallis tests, and the Kaplan-Meier survival and Cox proportional analysis where appropriate, using an SPSS (version 12) package.

\section{Results}

Distribution of ezrin, radixin and moesin family in mammary tissues. The three molecules were positively stained in 
A

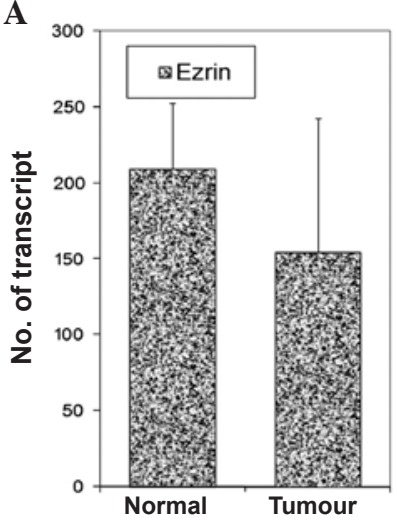

B

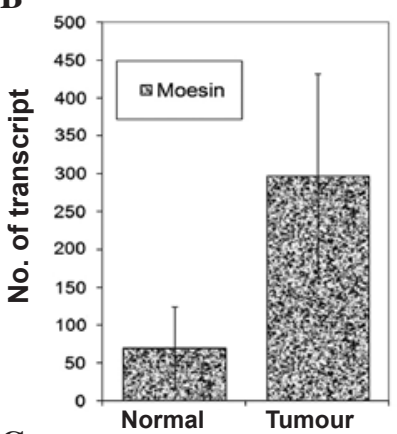

C

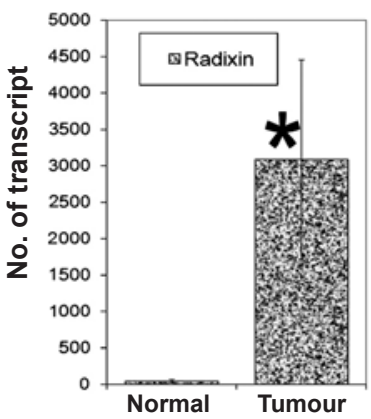

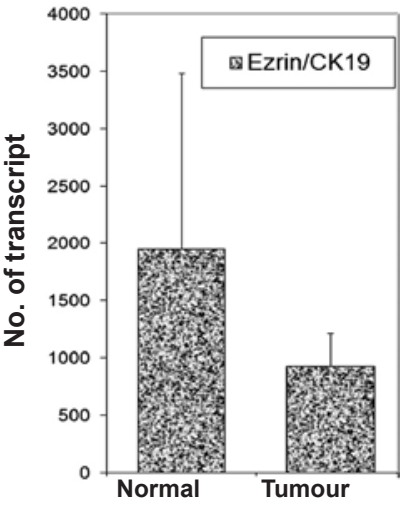
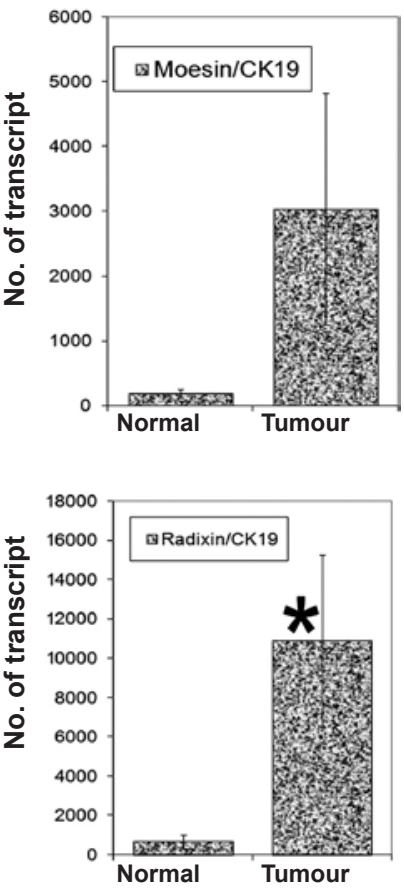

Figure 2. Levels of expression are shown in the left panel for (A) ezrin, (B) moesin and $(\mathrm{C})$ radixin in normal and tumour tissues. $\mathrm{p}<0.05$ compared with normal tissues. Right panel: ezrin, moesin and radixin CK19 ratios, respectively.

mammary tissues (Fig. 1). In normal tissues, ezrin prominently stained in epithelial cells. Its location is primarily on the luminal side and intercellular region of the epithelial cells. The luminal side of distribution is of particular interest, as ezrin is known to be rich in the microvilli area of the cells. The same distribution pattern was seen with radixin in normal mammary epithelial cells (Fig. 1, right panel), while that of moesin is less distinct.

Of note is that breast cancer cells have lost the staining pattern for the three molecules as was observed in normal epithelium.

Differential pattern of expression of the ezrin, radixin and moesin family in non-neoplastic and tumour tissues. Transcript levels of the three members were quantified using real-time quantitative PCR. As shown in Fig. 2, there were marginally low levels of ezrin $(\mathrm{p}=0.54)$ and high levels of moesin $(\mathrm{p}=0.12)$ in tumour tissues compared with normal tissues. A
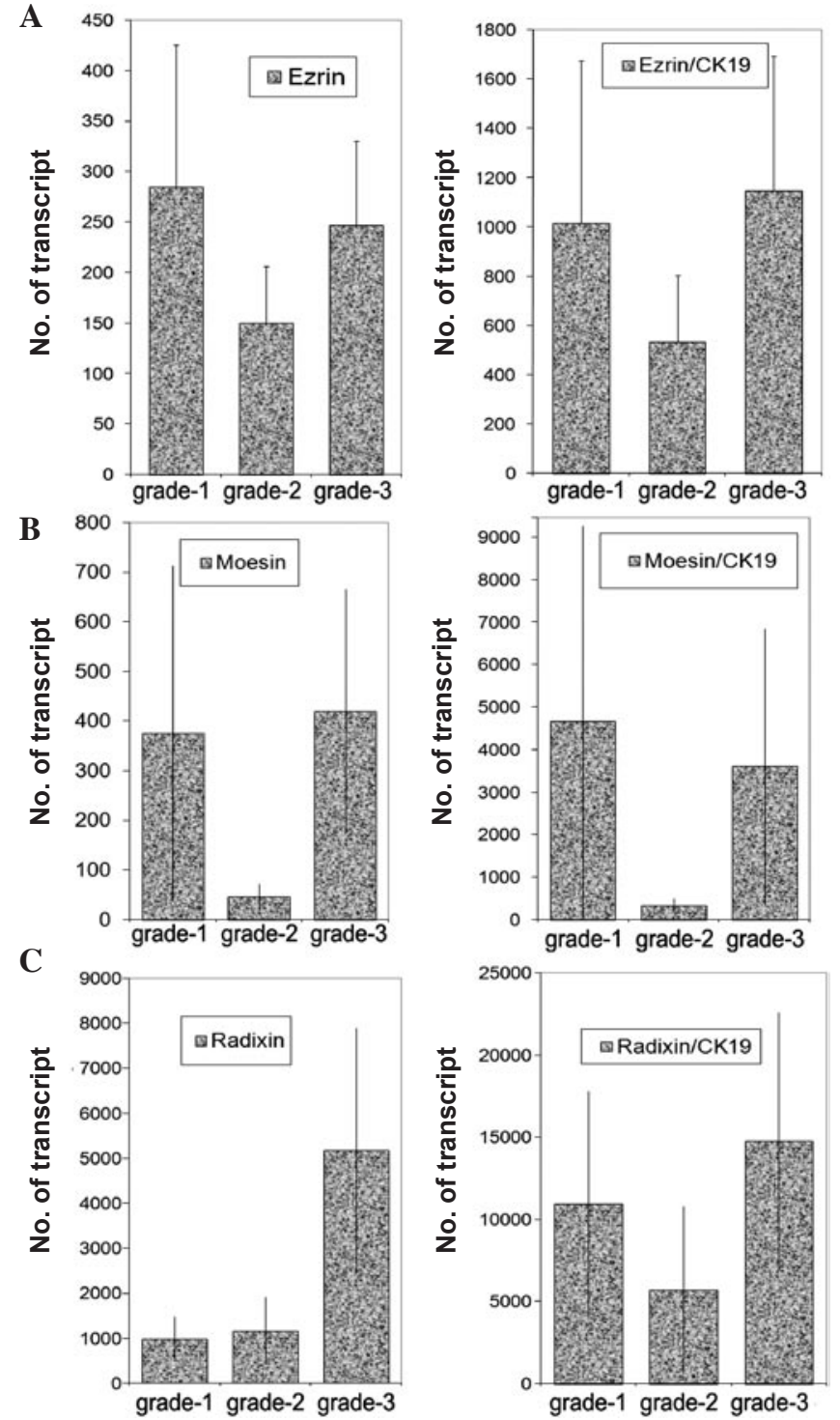

Figure 3. Levels of expression are shown in the left panel for (A) ezrin, (B) moesin and $(\mathrm{C})$ radixin in relation to the tumour grade. Right panel: respective ERM CK19 ratios.

similar trend was observed after normalisation with CK19 (Fig. 2, right panel). However, radixin transcript levels were significantly higher in tumour tissues $(\mathrm{p}=0.028)$, as was the radixin CK19 ratio (Fig. 2, right panel).

Correlation between ezrin, radixin and moesin proteins, nodal status and tumour grade. Transcript levels were analysed against tumour grade (Fig. 3), nodal status (Fig. 4) and predicted clinical prognosis, based on the Nottingham Prognostic Index (Fig. 5), but no significant difference was noted. However, there appear to be significant low levels of ezrin transcripts in TNM3 and TNM4 tumours ( $\mathrm{p}=0.0017$ and $\mathrm{p}=0.006$, respectively) (Fig. 6). No significant difference was noted for moesin and radixin.

Relationship between transcript levels of ezrin, radixin and moesin family members with clinical outcomes. As shown in Fig. 7, moesin and radixin levels together with their respec- 

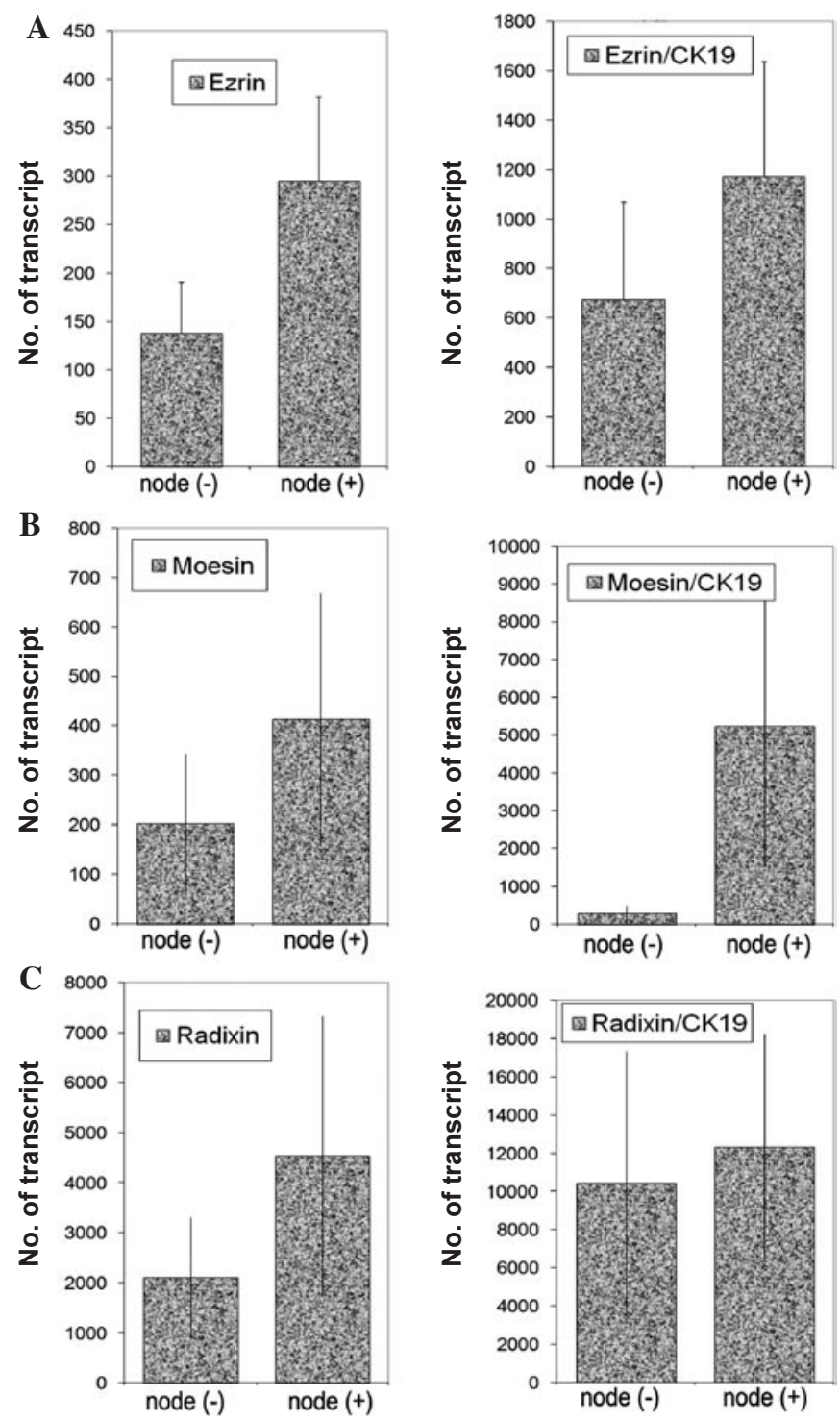

Figure 4. Levels of expression are shown in the left panel for (A) ezrin, (B) moesin and $(\mathrm{C})$ radixin in node-negative and node-positive tumours. Right panel: respective ERM CK19 ratios.

tive ratios to CK19 were inversely correlated with the patient outcome. Low levels of moesin and radixin transcripts and lower ratios to CK19 were noted in tumours obtained from patients with metastasis, local recurrence and in patients who succumbed to the disease (moesin: $\mathrm{p}=0.039, \mathrm{p}=0.037$ and $\mathrm{p}=0.066$, respectively, and radixin: $\mathrm{p}=0.039, \mathrm{p}=0.039$ and $\mathrm{p}=0.04$, respectively) (Fig. $7 \mathrm{~B}$ and $\mathrm{C}$ ). Although the levels of ezrin transcripts appear to be marginally higher in tumours associated with metastasis $(\mathrm{p}=0.53)$, the levels were significantly lower in tumour recurrence and in patients who succumbed to the disease $(\mathrm{p}=0.0001$ and $\mathrm{p}=0.59)$ (Fig. 7). Interestingly, the ezrin:CK19 ratio has shown a low level of survival in patients with metastasis, recurrence or mortality, compared with those who remained disease-free.

Using the Kaplan-Meier survival analysis, a general trend of higher levels of ERM was observed with marginal long overall (Fig. 8, left panel) and disease-free survival (Fig. 8, right panel). However, no statistical significance was noted.
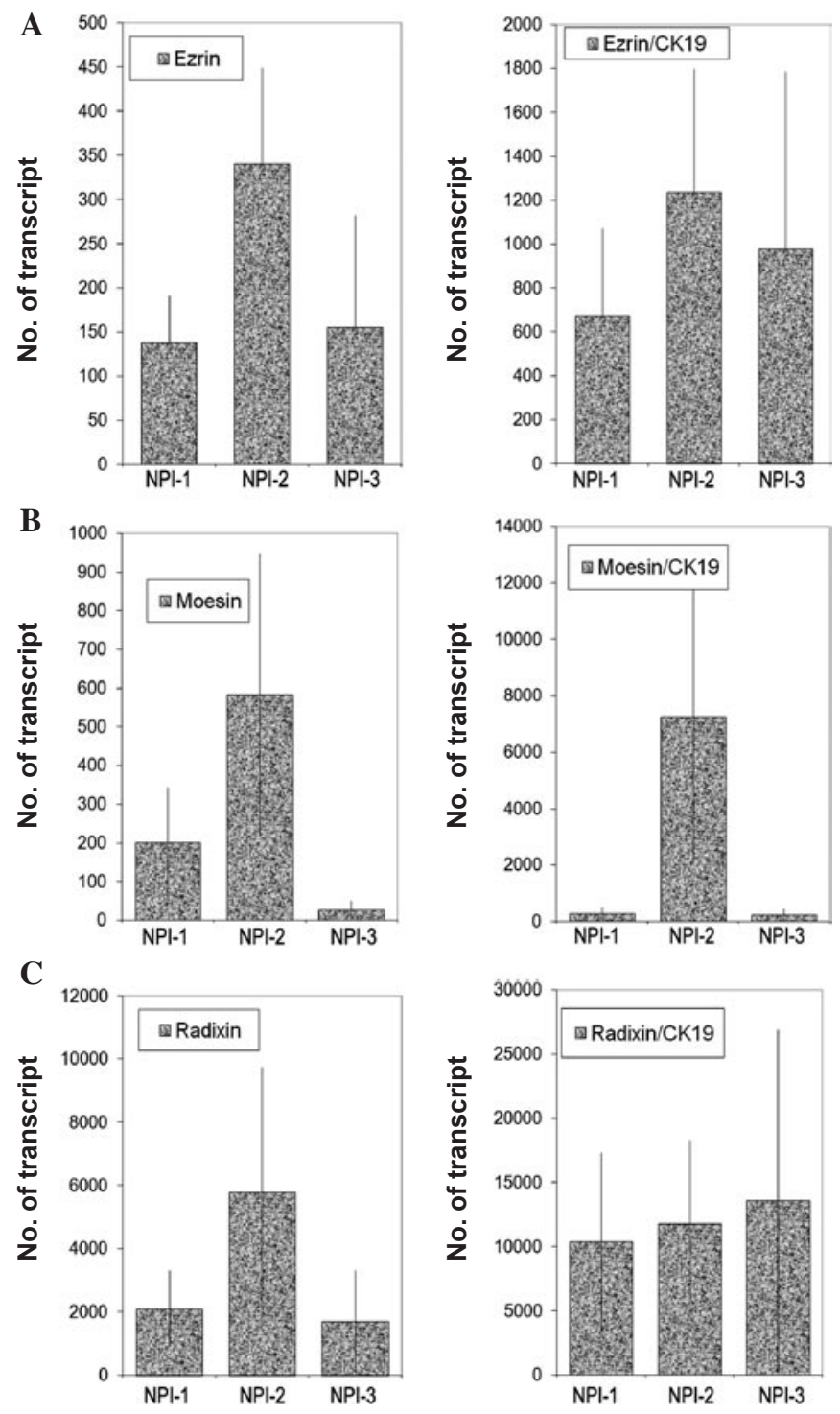

Figure 5. Expression of (A) ezrin, $(\mathrm{B})$ moesin and $(\mathrm{C})$ radixin in relation to the Nottingham Prognostic Index (NPI), where NPI-1 $(<3.4)$ indicates good prognosis, NPI-2 (3.4-5.4) moderate and NPI-3 (>5.4) poor prognosis. Right panel: respective ERM CK19 ratios.

\section{Discussion}

The present study is the first to report the association between the proteins belonging to the band 4.1 superfamily and breast cancer. The ERM subgroup of the superfamily has consistently been found to be co-expressed in the majority of cultured cells (1). In humans, the proteins have been found to have a tissuespecific expression pattern (1). High concentrations of ezrin are found in the lung, gut and the kidney while high levels of radixin occur in the liver and intestine (21). While endothelial cells express moesin, ezrin is expressed in epithelial and mesothelial cells (1). ERM molecules are found in high levels of actin-rich surface structures such as microvilli, filopodia and membrane ruffles (22). These are the vital structures required for the microinvasion and metastasis of cancer cells. The suppression of ERM expression by anti-sense oligonucleotides results in the destruction of the cell-substrate and cell-cell adhesion in colorectal cell lines (5) and thymoma 

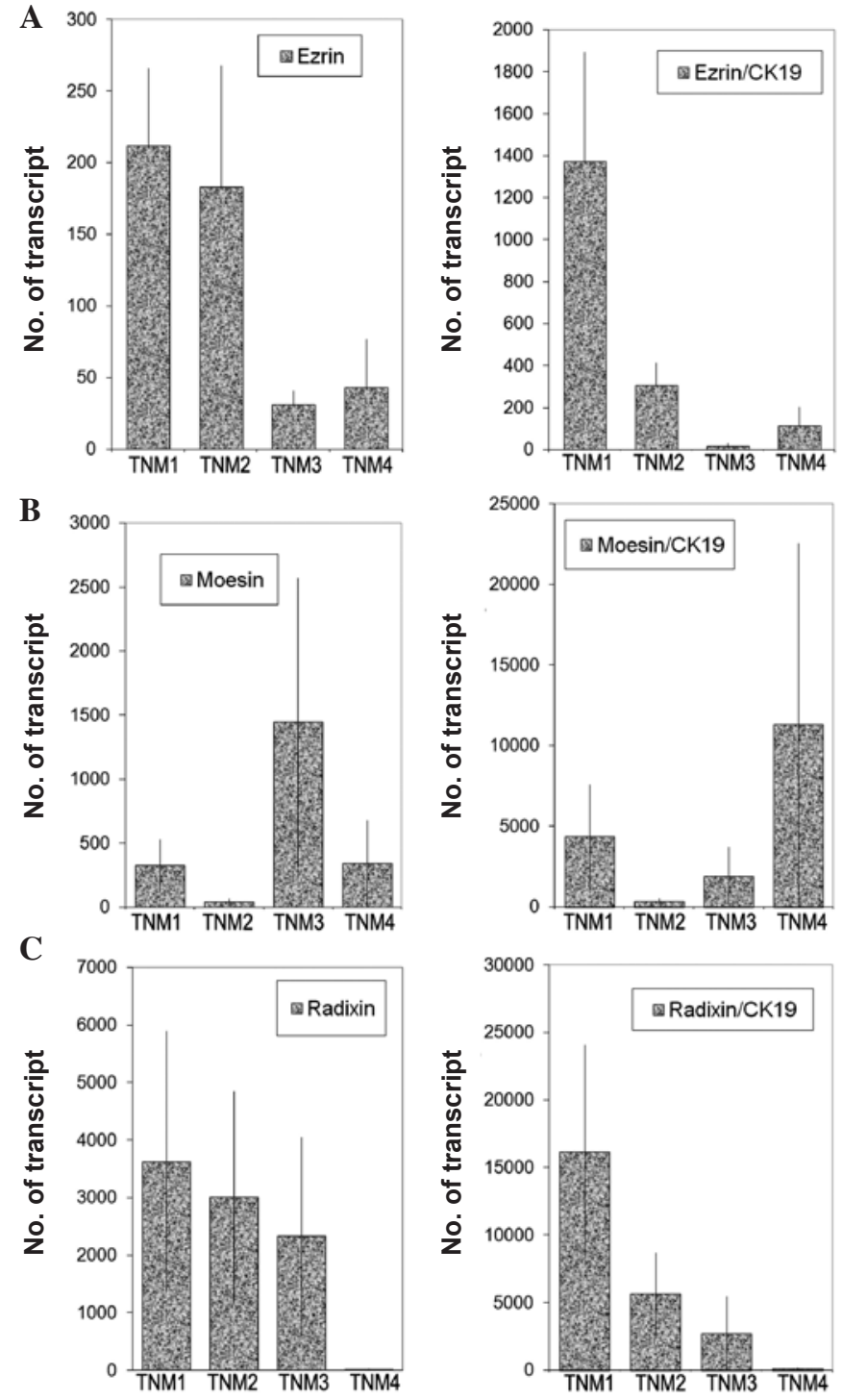

Figure 6. Levels of expression are shown in the left panel for (A) ezrin, (B) moesin and $(\mathrm{C})$ radixin in relation to TNM staging. Right panel: respective ERM CK19 ratios.

cells. Similarly it has been shown that the suppression of ERM expression resulted in the disappearance of microvilli from the cell surface (23). Therefore, if a link is found between the expression of these molecules and metastasis of breast cancer in patients, appropriate therapy may be employed to arrest the metastasis of the disease.

This study has revealed interesting associations between the ERM family and the clinical course of breast cancer. Such associations are observed between a higher incidence of recurrence and metastasis to low expression levels of the ERM family in the tumour tissues. The Kaplan-Meier analysis showed that a marginal overall improved survival occured in patients who had a higher expression of these proteins in their tumour tissue. Therefore, these results suggest an inverse relationship between ERM expression and survival benefit. We previously reported that following the inhibition of colorectal cancer cell lines using antisense nucleotides, these cells displayed a reduced cell-cell adhesion together with an
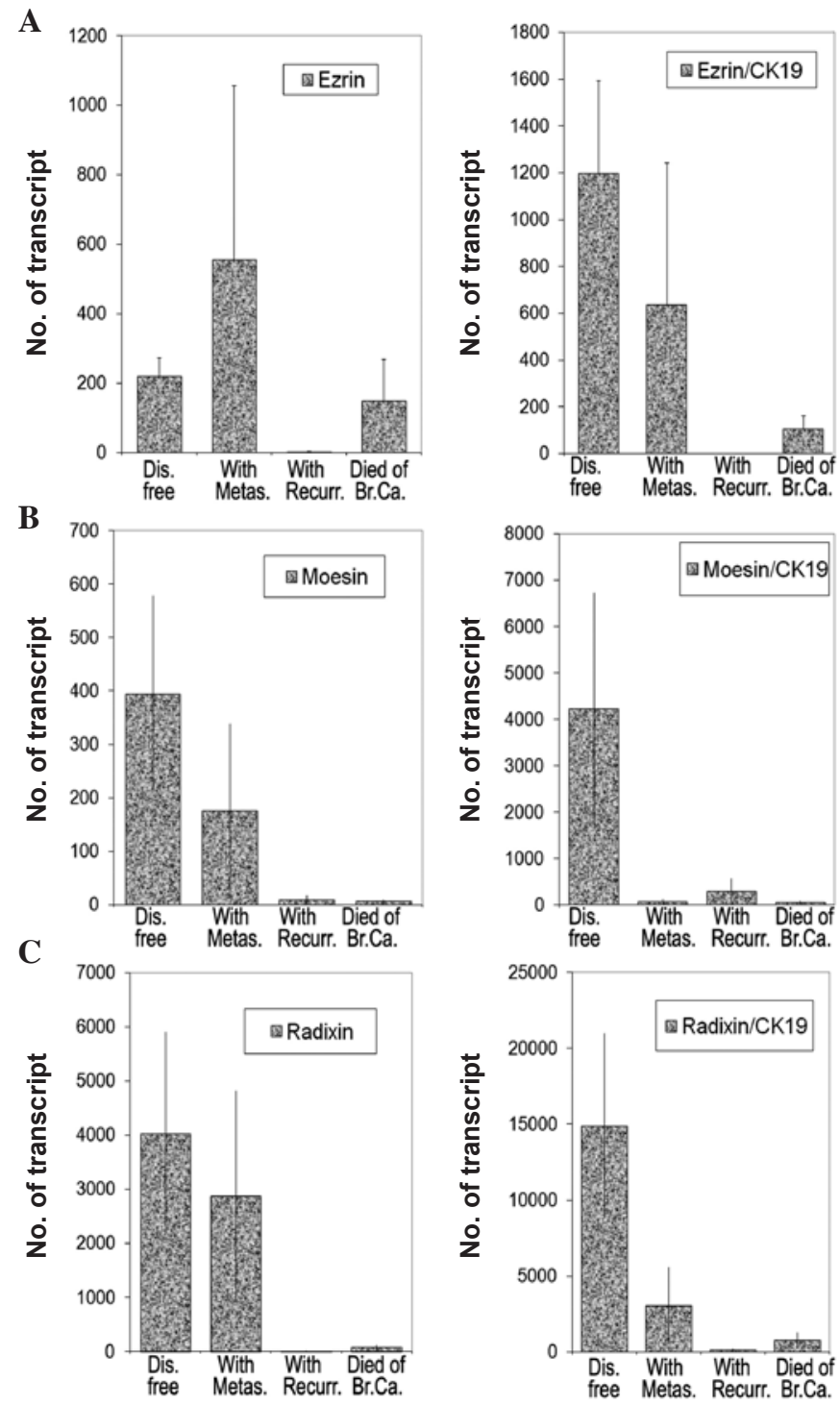

Figure 7. Levels of expression are shown in the left panel for (A) ezrin, (B) moesin and (C) radixin, as well as the clinical outcome over a 6-year follow-up period. The number of patients who remained disease-free, developed metastasis, had local recurrence and succumbed to the disease is shown. Right panel: respective ERM CK19 ratios.

increase in their motile and invasive behaviour. A correlation of the two studies shows that this mechanism also extends to breast cancer; thus, a higher incidence of recurrence and metastasis occurred in those cases with a low expression. Therefore, these results are important in predicting the tumour behaviour of these patients post-surgery.

The present study, together with that of Bruce et al (9) indicated that ezrin and other members of the ERM family are aberrant in their expression in breast cancer. This occurs at the protein [the present and Bruce et al (9)] and messenger level (the present study). A clinical connection exists since aberrant levels are associated with disease progression and clinical outcome. However, this connection appears to be weak in adenocarcinomas when compared to that observed in other tumour types, i.e. sarcomas and head and neck squamous cell carcinomas. Ezrin was found to be overexpressed in highgrade prostatic intraepithelial neoplasia (HGPIN) and prostate cancer compared with the adjacent benign prostatic epithelium. 
A

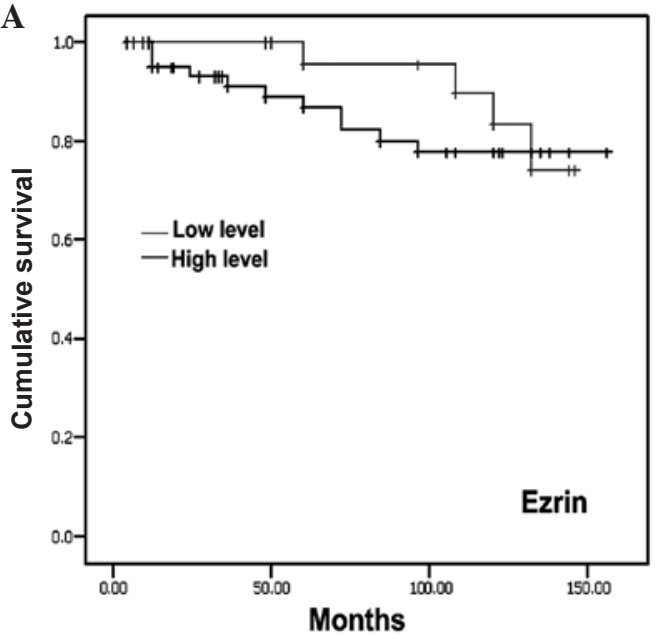

C

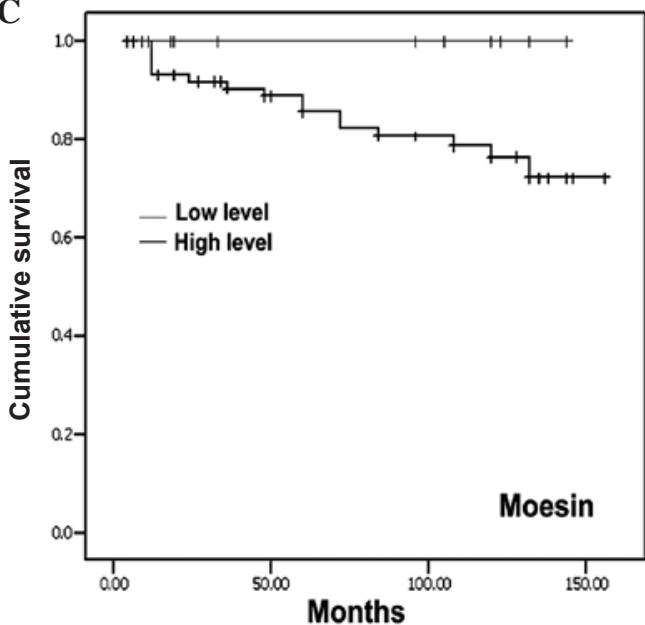

$\mathbf{E}$

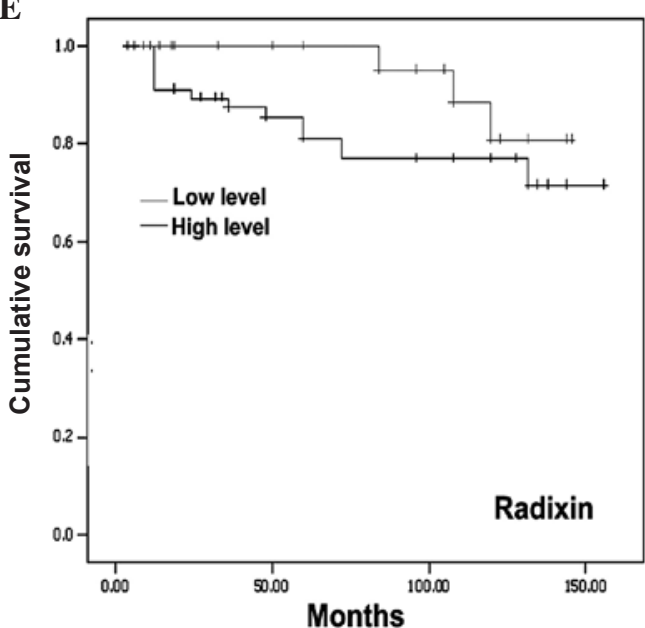

B

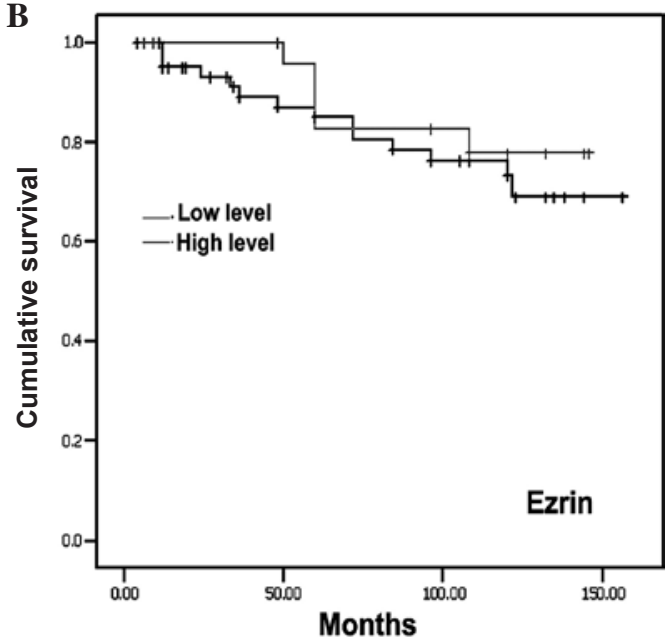

D

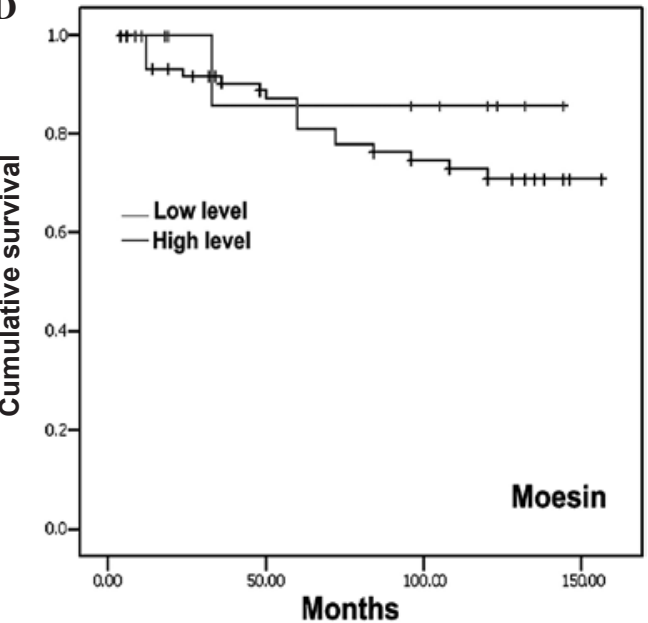

F

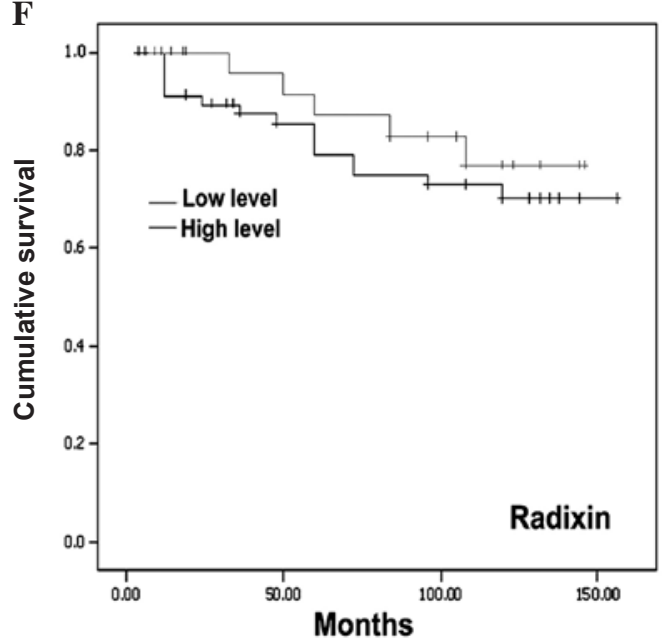

Figure 8. Levels of ERM family members and correlation with survival. Dark lines indicate low levels and grey lines indicate high levels of the respective family member.

In addition, HGPIN has a greater expression level of ezrin compared with that of prostate cancer. The above studies also indicated that the aberrant expression of ezrin is involved in the pathogenesis of prostate cancer; thus, ezrin expression may be useful for the diagnosis of HGPIN (7). Ezrin represents a promising therapeutic target for patients with advancedstage rhabdomyosarcoma (24). A high ezrin expression in the primary tumour of initially metastasis-free STS patients is strongly associated with the development of metastases during follow-up, and consequently with poor survival (8). A high ezrin expression in dog tumours was associated with early development of metastases. Consistent with these data, a significant association between a high ezrin expression and poor outcome was found in paediatric osteosarcoma patients (25). The expression pattern of ezrin in particular, and of the ERM family members in general, is contrary to the clinical pattern of disease progression in adenocarcinomas as compared to soft tissue sarcomas and other mesenchymal tumours. 
In conclusion, an inverse relationship between ERM expression and tumour behaviour of breast cancer patients was noted. Further studies, however, need to be conducted in other cancer types in clinical situations to obtain consistent results.

\section{Acknowledgements}

We would like to thank Cancer Research Wales for supporting this work.

\section{References}

1. Sato N, Funayama N, Nagafuchi A, Yonemura S and Tsukita S: A gene family consisting of ezrin, radixin and moesin. Its specific localization at actin filament/plasma membrane association sites. J Cell Sci 103: 131-143, 1992.

2. Pataky F, Pironkova R and Hudspeth AJ: Radixin is a constituent of stereocilia in hair cells. Proc Natl Acad Sci USA 101: 2601-2606, 2004

3. Faure S, Salazar-Fontana LI, Semichon M, Tybulewicz VL, Bismuth G, Trautmann A, Germain RN and Delon J: ERM proteins regulate cytoskeleton relaxation promoting T cell-APC conjugation. Nat Immunol 5: 272-279, 2004.

4. Vaheri A, Carpén O, Heiska L, Helander TS, Jääskeläinen J, Majander-Nordenswan P, Sainio M, Timonen T and Turunen O: The ezrin protein family: membrane-cytoskeleton interactions and disease associations. Curr Opin Cell Biol 9: 659-666, 1997.

5. Hiscox S and Jiang WG: Ezrin regulates cell-cell and cell-matrix adhesion, a possible role with E-cadherin//-catenin. J Cell Sci 112: 3081-3090, 1999.

6. Mhawech-Fauceglia P, Dulguerov P, Beck A, Bonet M and Allal AS: Value of ezrin, maspin and $\mathrm{nm} 23-\mathrm{H} 1$ protein expressions in predicting outcome of patients with head and neck squamouscell carcinoma treated with radical radiotherapy. J Clin Pathol 60: 185-189, 2007.

7. Valdman A, Fang X, Pang ST, Nilsson B, Ekman P and Egevad L: Ezrin expression in prostate cancer and benign prostatic tissue. Eur Urol 48: 852-857, 2005.

8. Weng WH, Ahlen J, Astrom K, Lui WO and Larsson C: Prognostic impact of immunohistochemical expression of ezrin in highly malignant soft tissue sarcomas. Clin Cancer Res 11: 6198-6204, 2005.

9. Bruce B, Khanna G, Ren L, Landberg G, Jirstrom K, Powell C, Borczuk A, Keller ET, Wojno KJ, Meltzer P, Baird K, McClatchey A, Bretscher A, Hewitt SM and Khanna C: Expression of the cytoskeleton linker protein ezrin in human cancers. Clin Exp Metastasis 24: 69-78, 2007.

10. Hanavadi S, Martin T, Mansel R and Jiang WG: Interleukin-11 and its receptor expression in human breast cancer. Ann Surg Oncol 13: 802-808, 2006.
11. Martin T, Goyal A, Mansel R, Watkins G and Jiang W: The transcription factor for the E-cadherin complex, Twist, Slug and Snail, in human breast cancer. Ann Surg Oncol 12: 1-9, 2005.

12. Jiang WG, Watkins G, Lane J, Cunnick G, Douglas-Jones A, Mokbel K and Mansel R: Prognostic value of Rho family and rho-GDIs in breast cancer. Clin Cancer Res 9: 6432-6440, 2003.

13. Jiang WG, Douglas-Jones A and Mansel R: Level of expression of PPAR-gamma and its co-activator (PPAR-GCA) in human breast cancer. Int J Cancer 106: 752-757, 2003.

14. Nazarenko A, Bhatnagar S and Hohman R: A closed tube format for amplification and detection of DNA based on energy transfer. Nucleic Acids Res 25: 2516-2521, 1997.

15. Parr C, Watkins G and Jiang WG: The possible correlation of Notch-1 and Notch-2 with clinical outcome and tumor clinicopathological parameters in human breast cancer. Int J Mol Med 14: 779-786, 2004.

16. Jiang WG, Grimshaw D, Lane J, Martin T, Abounader R, Laterra $\mathrm{J}$ and Mansel RE: A hammerhead ribozyme suppresses expresion of hepatocyte growth factor/scatter factor receptor c-MET and reduces migration and invasiveness of breast cancer cells. Clin Cancer Res 7: 2555-2562, 2001.

17. Ye L, Martin T, Parr C, Harrison G, Mansel R and Jiang WG: Biphasic effects of 17- $\beta$-oestradiol on expression of occludin and transendothelial resistance and paracellular permiability in human vascular endothelial cells. J Cell Physiol 196: 362-369, 2003.

18. King J, Ofori-Acquah SF, Stevens T, Al-Mehdi A-B, Fodstad O and Jiang WG: Activated leukocyte adhesion molecule in breast cancer: prognostic indicator. Breast Cancer Res 6: 478-487, 2004.

19. Davies G, Jiang WG and Mason MD: Cell-cell adhesion and signalling intermediates in human prostate cancer. J Urol 163: 985-992, 2000.

20. Berryman M, Franck Z and Bretscher A: Ezrin is concentrated in the apical microvilli of a wide variety of epithelial cells whereas moesin is found primarily in endothelial cells. J Cell Sci 105: 1025-1043, 1993.

21. Tsukita S and Hieda Y: A new 82-kD barbed end-capping protein (radixin) localized in the cell-to-cell adherens junction: purification and characterization. J Cell Biol 108: 2369-2382, 1989.

22. Yonemura S and Tsukita S: Direct involvement of ezrin/radixin/ moesin (ERM)-binding membrane proteins in the organization of microvilli in collaboration with activated ERM proteins. J Cell Biol 145: 1497-1509, 1999.

23. Takeuchi K, Sato N, Kasahara H, Funayama N, Nagafuchi A, Yonemura $S$ and Tsukita S: Perturbation of cell adhesion and microvilli formation by antisense oligonucleotides to ERM family members. J Cell Biol 125: 1371-1384, 1994.

24. Yu Y, Davicioni E, Triche TJ and Merlino G: The homeo-protein six 1 transcriptionally activates multiple protumorigenic genes but requires ezrin to promote metastasis. Cancer Res 66: 1982-1989, 2006.

25. Khanna C, Wan X, Bose S, Cassaday R, Olomu O, Mendoza A, Yeung C, Gorlick R, Hewitt SM and Helman LJ: The membranecytoskeleton linker ezrin is necessary for osteosarcoma metastasis. Nat Med 10: 182-186, 2004. 